\title{
A Concise Characterization of 3D Simple Points
}

\author{
Sébastien Fourey and Rémy Malgouyres \\ GREYC, ISMRA, 6 bd Maréchal Juin 14000 Caen - France \\ \{Fourey, Malgouyres\}@greyc.ismra.fr
}

\begin{abstract}
We recall the definition of simple points which uses the digital fundamental group introduced by T. Y. Kong in [Kon89]. Then, we prove that a not less restrictive definition can be given. Indeed, we prove that there is no need of considering the fundamental group of the complement of an object in order to characterize its simple points. In order to prove this result, we do not use the fact that "the number of holes of $X$ is equal to the number of holes in $\bar{X}$ " which is not sufficient for our purpose but we use the linking number defined in [FM00]. In so doing, we formalize the proofs of several results stated without proof in the literature (Bertrand, Kong, Morgenthaler).
\end{abstract}

Keywords: digital topology, linking number, simple points

\section{Introduction}

The definition of a simple point is the key notion in the context of thinning algorithms. Indeed, this definition leads to the most commonly admitted definition of the fact that a given thinning algorithm does preserve the topology of a digital image. Usually, one says that an image $\mathcal{I}_{1}$ is topologically equivalent to an image $\mathcal{I}_{2}$ if $\mathcal{I}_{1}$ can be obtained from $\mathcal{I}_{2}$ by sequential addition or deletion of simple points. In this case, a simple point is defined as a point the deletion of which does not change the topology of the image. The problem with topology preservation in $3 \mathrm{D}$ is that taking care not to change the number of connected components in the image as well as in its bakground is not sufficient as in 2D. In $3 \mathrm{D}$, one must also take care not to change the number and the location of the tunnels as donuts have. Now, different characterizations have been proposed by several authors which all lead to local characterizations which are equivalent. A first set of characterizations mainly use the Euler characteristic in order to count the number of tunnels, but even if this kind of characterization leads to a good local characterization it is limited by the fact that no localization of the tunnels is provided by the use the Euler characteristic (see Figure 3). Another definition has been proposed by Kong \& al. in [KR89] which is based on remarks made by Morgenthaler in [Mor81], with a new formalism which inlvolves the digital fundamental group introduced by Kong in [Kon89]. In this latter definition, topology preservation is expressed in term of existence of a canonical isomorphism between the fundamental group of the object and the fundamental group of the object without the considered point to be removed, and a similar 
isomorphism must exist in the background of the image (see Definition 3). In this paper, we prove that this second condition is in fact implied by the first one. In other words, we show that preserving the tunnels of the objects will imply the preservation of tunnels in the background. In order to prove that a such more concise characterization can be given, we use the linking number between paths of voxels as defined in [FM00] which provides an efficient way to prove that a given path cannot be homotopic to a degenerated path. Furtermore, the proof of Theorem 2 given in Section 3 uses propositions an lemmas, some of which are the direct answers to some open questions left by Morgenthaler in [Mor81] such as: do any two paths which can be be continuously deformed one into each other in an object $X$ keep this property after removal of a simple point of $X$ ?

\section{Definitions}

\subsection{Digital Image, Paths, Connectivity}

In this paper, we consider objects as subsets of the 3 dimensional space $\mathbb{Z}^{3}$. Elements of $\mathbb{Z}^{3}$ are called voxels (short for "volume elements"). The set of voxels which do not belong to an object $O \subset \mathbb{Z}^{3}$ constitute the complement of the object and is denoted by $\bar{O}$. Any voxel is identified with a unit cube centered at a point with integer coordinates $v=(i, j, k) \in \mathbb{Z}^{3}$. Now, we can define some binary symmetric and anti-reflexive relations between voxels. Two voxels are said 6-adjacent if they share a face, 18-adjacent if they share an edge and 26-adjacent if they share a vertex. By transitive closure of these adjacency relations, we can define another one: connectivity between voxels. We first define an $n$-path $\pi$ with a length $l$ from a voxel $a$ to a voxel $b$ in $O \subset \mathbb{Z}^{3}$ as a sequence of voxels $\left(y_{i}\right)_{i=0 \ldots l}$ such that for $0 \leq i<l$ the voxel $y_{i}$ is $n$-adjacent or equal to $y_{i+1}$, with $y_{0}=a$ and $y_{l}=b$. The path $\pi$ is a closed path if $y_{0}=y_{l}$ and is called a simple path if $y_{i} \neq y_{j}$ when $i \neq j$ (except for $y_{0}$ and $y_{l}$ if the path is closed). The voxels $y_{0}$ and $y_{l}$ are called the extremities of $\pi$ even in the case when the path is closed, and we denote by $\pi^{*}$ the set of voxels of $\pi$. A closed path $(x, x)$ with a length 1 for $x \in \mathbb{Z}^{3}$ is called a trivial path. If $x$ is a voxel of $\mathbb{Z}^{3}$ and $n \in\{6,18,26\}$ then we denote by $N_{n}(x)$ the set of voxels of $\mathbb{Z}^{3}$ which are $n$-adjacent to $x$. We call $N_{n}(x)$ the $n$-neighborhood of $x$. A subset $C$ of $\mathbb{Z}^{3}$ is called a simple closed $n$-curve if it is $n$-connected and any voxel of $C$ is $n$-adjacent to exactly two other voxels of $C$; then, if $c$ is a simple closed $n$-path such that $c^{*}=C, c$ is called a parameterized simple closed $n$-curve.

Given a path $\pi=\left(y_{k}\right)_{k=0, \ldots, l}$, we denote by $\pi^{-1}$ the sequence $\left(y_{k}^{\prime}\right)_{k=0, \ldots, l}$ such that $y_{k}=y_{l-k}^{\prime}$ for $k \in\{0, \ldots, l\}$.

Now we can define the connectivity relation: two voxels $a$ and $b$ are called $n$-connected in an object $O$ if there exists an $n$-path $\pi$ from $a$ to $b$ in $O$. This is an equivalence relation between voxels of $O$, and the $n$-connected components of an object $O$ are equivalence classes of voxels according to this relation.

In order to avoid topological paradoxes, we always study the topology of an object using an $n$-adjacency for the object and a complementary adjacency $\bar{n}$ for 
its complement. We sum up this by the use of a couple $(n, \bar{n})$ in $\{(6,26),(6+, 18)$, $(18,6+),(26,6)\}$. The notation $6+$ is used in order to distinguish the relation of 6 -connectivity associated to the 26 -connectivity from the $(6+)$-connectivity associated to the $18-$ connectivity.

If $\pi=\left(y_{i}\right)_{i=0, \ldots, p}$ and $\pi^{\prime}=\left(y_{k}^{\prime}\right)_{k=0, \ldots, p^{\prime}}$ are two $n$-paths such that $y_{p}=y_{0}^{\prime}$ then we denote by $\pi . \pi^{\prime}$ the path $\left(y_{0}, \ldots, y_{p-1}, y_{0}^{\prime}, \ldots, y_{p^{\prime}}^{\prime}\right)$ which is the concatenation of the two paths $\pi$ and $\pi^{\prime}$.

\subsection{Geodesic Neighborhoods and Topological Numbers}

The geodesic neighborhoods have been introduced by Bertrand ([Ber94]) in order to locally characterize in an efficient way the simple points of an object.

Definition 1 (geodesic neighborhood [Ber94]). Let $x \in X$, we define the geodesic neighborhood of $x$ in $X$ denoted by $G_{n}(x, X)$ as follows:

$-G_{6}(x, X)=\left(N_{6}(x) \cap X\right) \cup\left\{y \in N_{18}(x) \mid y\right.$ is 6-adjacent to a voxel of $\left.N_{6}(x) \cap X\right\}$.

- $G_{26}(x, X)=N_{26}(x) \cap X$.

Definition 2 (topological numbers [Ber94]). Let $X \subset \mathbb{Z}^{3}$ and $x \in \mathbb{Z}^{3}$. We define the topological number associated to $x$ and $X$, and we denote by $T_{n}(x, X)$ for $(n, \bar{n}) \in\{(6,26),(26,6)\}$, the number of connected components of $G_{n}(x, X)$.

\subsection{Digital Fundamental Group}

In this section, we define the digital fundamental group of a subset $X$ of $\mathbb{Z}^{3}$ following the definition of Kong in [Kon89] and [Kon88].

First, we need to introduce the $n$-homotopy relation between $n$-paths in $X$. Intuitively, a path $c$ is homotopic to a path $c^{\prime}$ if $c$ can be "continuously deformed" into $c^{\prime}$. Let us consider $X \subset \mathbb{Z}^{3}$. First, we introduce the notion of an elementary $n$-deformation. Two closed $n$-paths $c$ and $c^{\prime}$ in $X$ having the same extremities are the same up to an elementary $n$-deformation (with fixed extremities) in $X$, and we denote $c \sim_{n} c^{\prime}$, if they are of the form $c=\pi_{1} \cdot \gamma \cdot \pi_{2}$ and $c^{\prime}=\pi_{1} \cdot \gamma^{\prime} \cdot \pi_{2}$, the $n$-paths $\gamma$ and $\gamma^{\prime}$ having the same extremities and being both included in a $2 \times 2 \times 2$ cube if $(n, \bar{n})=(26,6)$, in a $2 \times 2$ square if $(n, \bar{n})=(6,26)$. Now, the two $n$-paths $c$ and $c^{\prime}$ are said to be $n$-homotopic (with fixed extremities) in $X$ if there exists a finite sequence of $n$-paths $c=c_{0}, \ldots, c_{m}=c^{\prime}$ such that for $i=0, \ldots, m-1$ the $n$-paths $c_{i}$ and $c_{i+1}$ are the same up to an elementary $n$-deformation (with fixed extremities). In this case, we denote $c \simeq_{n} c^{\prime}$. A closed $n$-path $c=\left(x_{0}, \ldots, x_{q}=x_{0}\right)$ in $X$ is said to be $n$-reducible in $X$ if $c \simeq_{n}\left(x_{0}, x_{0}\right)$ in $X$.

Let $B \in X$ be a fixed voxel of $X$ called the base surfel. We denote by $A_{B}^{n}(X)$ the set of all closed $n$-paths $c=\left(x_{0}, \ldots, x_{p}\right)$ which are included in $X$ and such that $x_{0}=x_{p}=B$. The $n$-homotopy relation is an equivalence relation on $A_{B}^{n}(X)$, and we denote by $\Pi_{1}^{n}(X, B)$ the set of the equivalence classes of this equivalence relation. If $c \in A_{B}^{n}(X)$, we denote by $[c]_{\Pi_{1}^{n}(X, B)}$ the equivalence class of $c$ under this relation. 
The concatenation of closed $n$-paths is compatible with the $n$-homotopy relation, hence it defines an operation on $\Pi_{1}^{n}(X, B)$, which to the class of $c_{1}$ and the class of $c_{2}$ associates the class of $c_{1} \cdot c_{2}$. This operation provides $\Pi_{1}^{n}(X, B)$ with a group structure. We call this group the $n$-fundamental group of $X$. The $n$-fundamental group defined using a voxel $B^{\prime}$ as base surfel is isomorphic to the $n$-fundamental group defined using a voxel $B$ as base surfel if $X$ is $n$-connected.

Now, we consider $Y \subset X \subset \mathbb{Z}^{3}$ and $B \in Y$ a base voxel. A closed $n$-path in $Y$ is a particular case of a closed $n$-path in $X$. Furthermore, if two closed $n$-paths of $Y$ are $n$-homotopic (with fixed extremities) in $Y$, they are $n$-homotopic (with fixed extremities) in $X$. These two properties enable us to define a canonical morphism $i_{*}: \Pi_{1}^{n}(Y, B) \longrightarrow \Pi_{1}^{n}(X, B)$, which we call the morphism induced by the inclusion map $i: Y \longrightarrow X$. To the class of a closed $n$-path $\alpha \in A_{B}^{n}(Y)$ in $\Pi_{1}^{n}(Y, B)$ the morphism $i_{*}$ associates the class of the same $n$-path in $\Pi_{1}^{n}(X, B)$.

\subsection{The Digital Linking Number}

The digital linking number, denoted by $L_{\pi, c}$, has been defined in [FM00] for a couple $(\pi, c)$ of closed paths of $\mathbb{Z}^{3}$ which do not intersect each other. It is the digital analogue of the linking number defined in knot theory (see [Rol]) and it is immediately computable (see [FM00]) for any couple $(\pi, c)$ such that $\pi$ is an $n$-path and $c$ is an $\bar{n}$-path with $(n, \bar{n}) \in\{(6,26),(26,6),(6+, 18),(18,6+)\}$. This number counts the number of times two digital closed paths are interlaced one with each other, as illustrated in Figure 1. Since the precise definition of the linking number is too long to be recalled here, we simply give the idea of the way it can be computed in Figure 2. In this figure, we have depicted several configurations which illustrate the contributions of superposed voxels, one in each paths, in a $2 \mathrm{D}$ projection of the two paths. The digital linking number is nothing but the sum of such contributions for all couples of overlapping voxels.

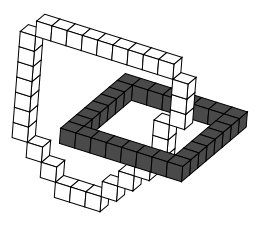

(a) $L_{\pi, c}= \pm 1$.

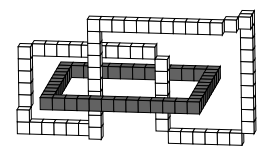

(b) $L_{\pi, c}= \pm 2$.

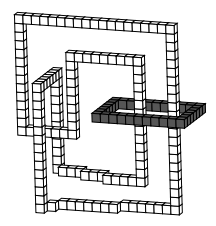

(c) The Whitehead's link: $L_{\pi, c}=0$.

Fig. 1. Three kinds of links: a 6 -path $\pi$ in black and a $18-$ path $c$ in white

The two following theorems have been proved in [FM00] and allows to say that the linking number is a new topological invariant in the field of digital topology. 


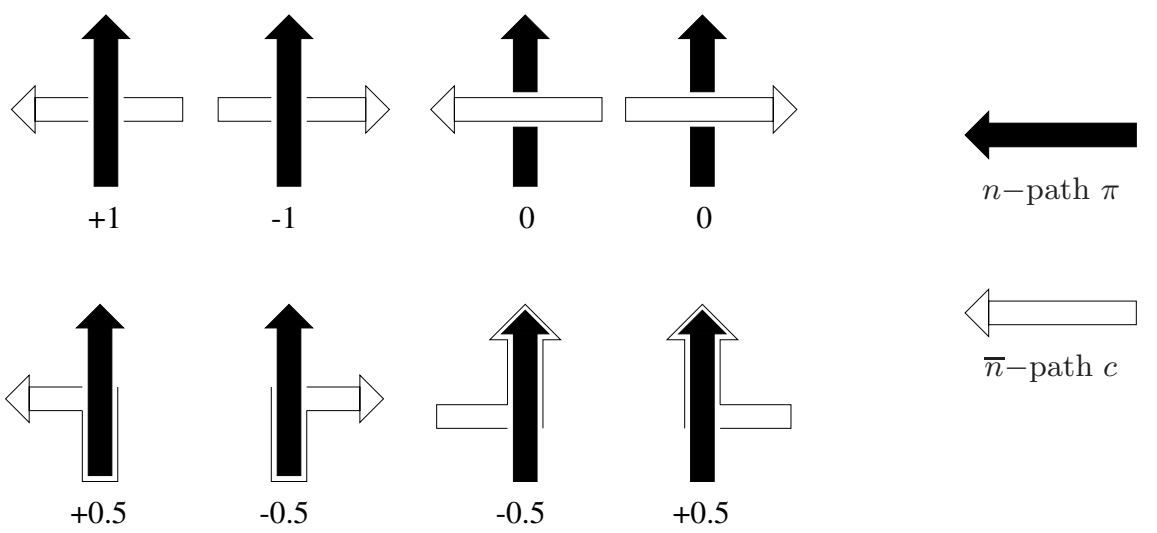

Fig. 2. Contributions associated with voxels where the two paths of a link overlap in a $2 \mathrm{D}$ projection of a link

Theorem 1. Let $\pi$ and $\pi^{\prime}$ be two closed $n$-path and $c$ be a closed $\bar{n}$-path of $\mathbb{Z}^{3}$ such that $\pi^{*} \cap c^{*}=\emptyset$ and $\pi^{\prime *} \cap c^{*}=\emptyset$. If $\pi \simeq_{n} \pi^{\prime}$ in $\mathbb{Z}^{3} \backslash c^{*}$ then $L_{\pi, c}=L_{\pi^{\prime}, c}$ and $L_{c, \pi}=L_{c, \pi^{\prime}}$.

Remark 1. If $c$ is a trivial path, then $L_{c, \pi}=0$ for any closed $n$-path such that $c^{*} \cap \pi^{*}=\emptyset$. It follows that if a closed $n$-path $c$ in $X \subset \mathbb{Z}^{3}$ is $n$-reducible in $X$, then $L_{c, \pi}=0$ for all closed $\bar{n}$-path $\pi$ in $\overline{c^{*}}$.

\subsection{Characterization of Simple Points}

A simple point for $X \subset \mathbb{Z}^{3}$ is a point the deletion of which does not change the topology of $X$. Now, topology preservation in 3D is not as simple to express as in the $2 \mathrm{D}$ case because of the existence of tunnels. A few authors have used two main tools to study topology preservation: the Euler characteristic which only allows to count the number of tunnels of an object ([TF82]), and the digital fundamental group ([Kon89]) which allows to "localize" the tunnels. Indeed, as depicted in Figure 3, counting the number of tunnels is not sufficient to characterize the fact that the topology is preserved. In this paper, we are interested by a definition of simple points which uses the digital fundamental group and which avoids the problem previously mentioned. The following definition appears as the most convenient for the property "the deletion of $x$ preserves topology of $X$ ". It comes from the criterion given in [Kon89] for a thinning algorithm to preserve topology.

Definition 3. Let $X \subset \mathbb{Z}^{3}$ and $x \in X$. The point $x$ is said to be $n$-simple if:

i) $X$ and $X \backslash\{x\}$ have the same number of $n$-connected components.

ii) $\bar{X}$ and $\bar{X} \cup\{x\}$ have the same number of $\bar{n}$-connected components.

iii) For each voxel $B$ in $X \backslash\{x\}$, the group morphism $i_{*}: \Pi_{1}^{n}(X \backslash\{x\}, B) \longrightarrow$ $\Pi_{1}^{n}(X, B)$ induced by the inclusion map $i: X \backslash\{x\} \longrightarrow X$ is an isomorphism. 

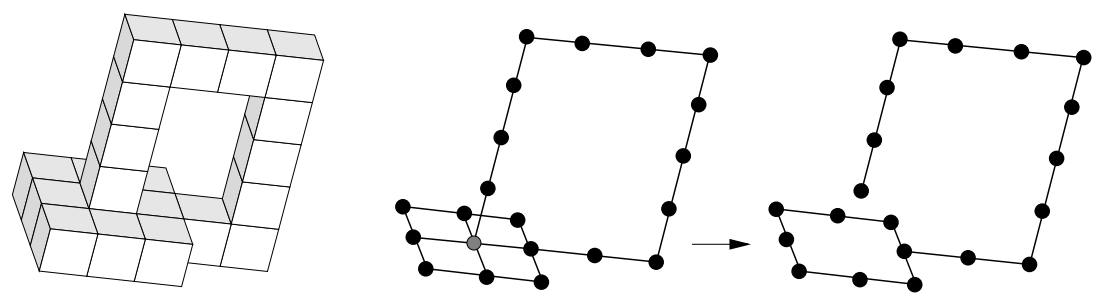

Fig. 3. The gray point can be removed without changing the Euler characteristic of the object which is equal to zero. However, this point is obviously not $n$-simple (if $(n, \bar{n}) \in\{(6,18),(6,26)\})$

iv) For each voxel $B^{\prime}$ in $\bar{X}$, the group morphism $i_{*}^{\prime}: \Pi_{1}^{\bar{n}}\left(X, B^{\prime}\right) \bar{X} \longrightarrow \Pi_{1}^{\bar{n}}(\bar{X} \cup$ $\left.\{x\}, B^{\prime}\right)$ induced by the inclusion map $i^{\prime}: \bar{X} \longrightarrow \bar{X} \cup\{x\}$ is an isomorphism.

Bertrand, in [BM94], gave a local characterization for 3D simple points in term of number of connected components in geodesic neighborhoods. However, the definition of simple point given in [BM94] differs from the definition used here since it does not consider any morphism between digital fundamental groups but only require the preservation of cavities and "tunnels". One purpose of this paper is to well formalize the fact that the local characterization given by Bertrand is a consequence of the three first conditions of Definition 3 and conversly that the four conditions are themselves consequences of the local characterization using the topological numbers. We recall here the characterization given by Bertrand and Malandain in [BM94]. Note that the definition of simple points used in this proposition slightly differs from Definition 3.

Proposition 1 ([BM94]). Let $x \in X$ and $(n, \bar{n}) \in\{(6,26),(26,6)\}$. The point $x$ is a $n$-simple point if and only if $T_{n}(x, X)=1$ and $T_{\bar{n}}(x, \bar{X})=1$.

\section{A New Characterization of 3D Simple Points}

In the sequel of this paper $(n, \bar{n}) \in\{(6,26),(26,6)\}$.

In this section, we state the main result of this paper which is that a not less restrictive criterion for topology preservation is obtained using the only conditions $i$ ), $i$ ) and $i i i$ ) of Definition 3. In other words, we prove the following theorem:

Theorem 2. Let $X \subset \mathbb{Z}^{3}$ and $x \in X$. The point $x$ is $n$-simple for $X$ if and only if:

i) $X$ and $X \backslash\{x\}$ have the same number of connected components.

ii) $\bar{X}$ and $\bar{X} \cup\{x\}$ have the same number of connected components.

iii) For each voxel $B$ in $X \backslash\{x\}$, the group morphism $i_{*}: \Pi_{1}^{n}(X \backslash\{x\}, B) \longrightarrow$ $\Pi_{1}^{n}(X, B)$ induced by the inclusion map $i: X \backslash\{x\} \longrightarrow X$ is an isomorphism. 
In order to prove this theorem, we first prove (Subsection 3.1) that a voxel which satisfies the three conditions of Theorem 2 also satisfies the the local characterization given by Proposition 1 and then, we show (Subsection 3.2) that this characterization itself implies that the four conditions of Definition 3 are satisfied.

In the sequel of the paper, we may suppose without loss of generality that $X$ is an $n$-connected subset of $\mathbb{Z}^{3}$; and that $x$ and $B$ are two distinct voxels of $X$ whereas $B^{\prime}$ is a voxel of $\bar{X}$. Furthermore, $i_{*}: \Pi_{1}^{n}(X \backslash\{x\}, B) \longrightarrow \Pi_{1}^{n}(X, B)$ is the group morphism induced by the inclusion of $X \backslash\{x\}$ in $X$; and $i_{*}^{\prime}: \Pi_{1}^{\bar{n}}\left(\bar{X}, B^{\prime}\right) \longrightarrow$ $\Pi_{1}^{\bar{n}}\left(\bar{X} \cup\{x\}, B^{\prime}\right)$ is the group morphism induced by the inclusion of $\bar{X}$ in $\bar{X} \cup\{x\}$.

Remark 2. In the sequel, we shall admit the basic property that, if $Y \subset X$ are $n$-connected subsets of $\mathbb{Z}^{3}$, the group morphism from $\Pi_{1}^{n}(Y, B)$ to $\Pi_{1}^{n}(X, B)$ induced by the inclusion of $Y$ in $X$ for a base surfel $B \in Y$ is an isomorphism if and only if the group morphism between $\Pi_{1}^{n}\left(Y, B^{\prime}\right)$ and $\Pi_{1}^{n}\left(X, B^{\prime}\right)$ is an isomorphism for any base surfel $B^{\prime} \in Y$.

\subsection{First Step of the Proof of Theorem 2}

The purpose of this section is to prove the following proposition.

Proposition 2. If the conditions $i$ ), ii) and iii) of Definition 3 are satisfied, then $T_{n}(x, X)=1$ and $T_{\bar{n}}(x, \bar{X})=1$.

In order to prove this proposition, we introduce several other propositions and lemmas.

Proposition 3. If $T_{n}(x, X) \geq 2$, then either an $n$-connected component of $X$ is created by deletion of $x$, or the morphism $i_{*}$ is not onto.

Sketch of proof of Proposition 3: The proof of a similar proposition given in [BM94] may be adapted to our formalism. It involves the definition of a number $\nu(x, \alpha, C)$ which counts the number of time a given $n$-path $\alpha$ goes from a component $C$ of $G_{n}(x, X)$ to $x$ minus the number of times $\alpha$ goes from $x$ to $C$ (see Figure 4(a)). This number is shown to be invariant under $n$-homotopic deformations of the $n$-path $\alpha$ inside $X$. Then, it allows to prove that, when connected components of $G_{n}(x, X)$ are $n$-connected in $X \backslash\{x\}$, an homotopy class of paths distinct from the class of the trivial path, cannot be reached by the morphism $i_{*}$ (class of the path $\alpha$ of Figure 4(a)).

Here, we state the following new proposition.

Proposition 4. If $T_{n}(x, X)=1$ and $T_{\bar{n}}(x, \bar{X}) \geq 2$ then two $\bar{n}$-connected component of $\bar{X}$ are merged by deletion of $x$ or the morphism $i_{*}$ is not one to one.

The main idea of this paper is to use the linking number in order to prove Proposition 4. Indeed, until this paper and the possible use of the linking number, one could prove that when $T_{n}(x, X)=1$ and $T_{\bar{n}}(x, \bar{X}) \geq 2$ and no $\bar{n}$-connected 


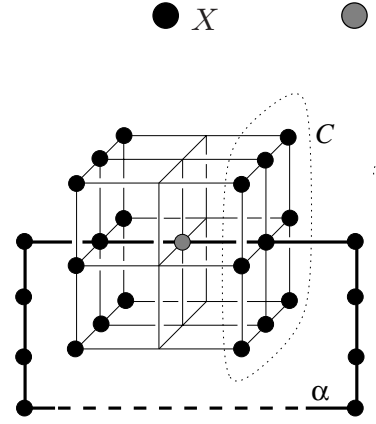

(a) $\nu(x, \alpha, C)= \pm 1$

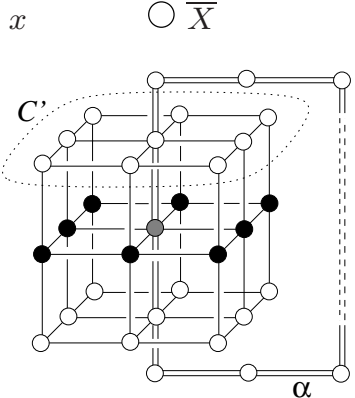

(b) $\nu\left(x, \alpha, C^{\prime}\right)= \pm 1$

Fig. 4. Parts of the proofs of Propositions 3 and 5

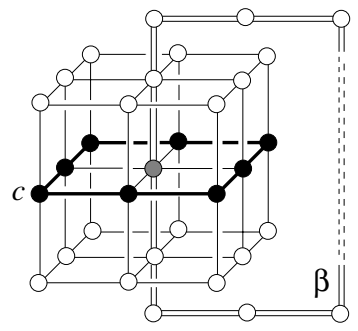

$L_{c, \beta}= \pm 1$

Fig. 5. Idea of the proof of Proposition 4

component of $\bar{X}$ are merged by deletion of $x$, then, the morphism $i_{*}{ }^{\prime}$ induced by the inclusion of $\bar{X}$ in $\bar{X} \cup\{x\}$ is not onto. In other words, "a hole is created in $\bar{X} \cup\{x\}$ ". Indeed, a similar proof to Proposition 3 would lead to the following proposition (see Figure 4(b)).

Proposition 5. If $T_{n}(x, X)=1$ and $T_{\bar{n}}(x, \bar{X}) \geq 2$ then two $\bar{n}$-connected component of $\bar{X}$ are merged by deletion of $x$ or the morphism $i_{*}^{\prime}$ is not onto.

In this paper, we show that in this case "a hole is created in $X \backslash\{x\}$ " or more formally, $i_{*}$ is not one to one. This is proved using the linking number as illustrated in Figure 5 where the closed path $c$ is reducible in $X$ (Lemma 2 below) whereas it is not reducible in $X \backslash\{x\}$ since $L_{c, \beta}= \pm 1$ (Remark 1). This shows that a condition on the preservation of tunnels in the object (Condition iii of Definition 3) is sufficient to ensure that tunnels of the complement are also left unchanged. And the proof of this result is obtained with the only formalism provided by the use of the digital fundamental group.

Before proving Proposition 4, we must state the two following lemmas.

Lemma 1. Let $x \in X$ such that $T_{n}(x, X)=1$ and $T_{\bar{n}}(x, \bar{X}) \geq 2$ and let $A=$ $G_{n}(x, X)$. Then there exists a parameterized simple closed $n$-curve $c$ in $A$ (see Figure 5) and a closed $\bar{n}-$ path $\beta$ in $\overline{N_{26}(x) \cap X}$ such that $L_{c, \beta}= \pm 1$.

The latter lemma has been proved by investigating, using a computer, all the possible local configurations in the neighborhood of a voxel $x$ such that $T_{n}(x, X)=1$ and $T_{\bar{n}}(x, \bar{X}) \geq 2$; showing for each one the existence of a simple closed curve in $G_{n}(x, X)$. The existence of the $\bar{n}$-path $\beta$ of Lemma 1 is then proved using local considerations.

Lemma 2. Let $x$ be a point of $X$ such that $T_{n}(x, X)=1$. Then, any closed $n$-path c in $G_{n}(x, X)$ is $n$-reducible in $X$. 
Sketch of proof of Proposition 4: Let $x$ be a point of $X$ such that $T_{n}(x, X)=$ 1 and $T_{\bar{n}}(x, \bar{X}) \geq 2$. Let $c$ and $\beta$ be the paths of Lemma 1 ; and let $a$ and $b$ be the two voxels of $\beta$ which are $\bar{n}$-adjacent to $x$. If $a$ and $b$ are not $\bar{n}$-connected in $\bar{X}$ then it is clear that two $\bar{n}$-connected components of $\bar{X}$ are merged by deletion of $x$ from $X$.

If $a$ and $b$ are connected by an $\bar{n}$-path $\alpha^{\prime}$ in $\bar{X}$. Then, it follows that $\beta$ is $\bar{n}$-homotopic to the path $\alpha=(a) \cdot \alpha^{\prime} .(b, x, a)$ in $\overline{\left(N_{26}(x) \cap X\right)}$. Now, from Theorem 1 and since $\overline{\left(N_{26}(x) \cap X\right)} \subset \overline{c^{*}}$ we have $L_{c, \beta}=L_{c, \alpha}= \pm 1$.

From Theorem 1 (and Remark 1), it follows that the path $c$ is not $n$-reducible in $\overline{\alpha^{\prime *}}$ and since $\alpha^{*} \subset \bar{X} \cup\{x\}$ then $X \backslash\{x\} \subset \overline{\alpha^{*}}$ so that a fortiori $\alpha$ cannot be $n$-reducible in $X \backslash\{x\}$. Formally, if $c$ is a closed path from a voxel $A$ to $A$ in $X \backslash$ $\{x\}$, we have $[c]_{\Pi_{1}^{n}(X \backslash\{x\}, A)} \neq[1]_{\Pi_{1}^{n}(X \backslash\{x\}, A)}$. Now, from Lemma $2, c \simeq_{n}(A, A)$ in $X$ so that $i_{*}\left([c]_{\Pi_{1}^{n}(X \backslash\{x\}, A)}\right)=[c]_{\Pi_{1}^{n}(X, A)}=[1]_{\Pi_{1}^{n}(X, A)}=i_{*}\left([1]_{\Pi_{1}^{n}(X \backslash\{x\}, A)}\right)$, i.e. $i_{*}$ is not one to one.

Proof of Proposition 2: Suppose that properties $i$ ), ii) and iii) of Definition 3 are satisfied. From Proposition 3 we deduce that $T_{n}(x, X)<2$. Furthermore, if no connected component of $X$ is removed then $T_{n}(x, X) \neq 0$. Finally, $T_{n}(x, X)=1$.

From Proposition 4 we deduce that $T_{\bar{n}}(x, \bar{X})<2$. Then, if no connected component of $\bar{X}$ is created then $T_{\bar{n}}(x, \bar{X}) \neq 0$. Finally, $T_{\bar{n}}(x, \bar{X})=1$.

\subsection{Second Step of the Proof of Theorem 2}

We prove the following proposition.

Proposition 6. If $T_{n}(x, X)=1$ and $T_{\bar{n}}(x, \bar{X})=1$, then conditions $\left.i\right)$, ii), iii) and $i v)$ of Definition 3 are satified.

The main difficult part of the proof of Proposition 6 relies on the following proposition.

Proposition 7. If $T_{n}(x, X)=1$ and $T_{\bar{n}}(x, \bar{X})=1$ then $i_{*}$ is an isomorphism.

Corollary 1. If $T_{n}(x, X)=1$ and $T_{\bar{n}}(x, \bar{X})=1$ then $i_{*}^{\prime}$ is an isomorphism.

In order to prove Proposition 7 we first state Lemma 3 and Lemma 4.

Lemma 3. If $T_{n}(x, X)=1$ and $T_{\bar{n}}(x, \bar{X})=1$ then for all $B \in X \backslash\{x\}$ and all $n$-path c of $A_{n}^{B}(X)$, there exists a path $c^{\prime}$ in $A_{n}^{B}(X \backslash\{x\})$ such that $c \simeq_{n} c^{\prime}$ in $X$.

Lemma 4. If $T_{n}(x, X)=1$ and $T_{\bar{n}}(x, \bar{X})=1$ then, for any voxel $B \in X \backslash\{x\}$, two closed $n$-paths $c$ and $c^{\prime}$ of $A_{n}^{B}(X \backslash\{x\})$ which are $n$-homotopic in $X$ are $n$-homotopic in $X \backslash\{x\}$.

Sketch of proof of Proposition 7: Lemma 3 allows to prove that $i_{*}$ is onto, and Lemma 4 allows to prove that $i_{*}$ is one to one.

Proof of Proposition 6: It is readily seen that $T_{n}(x, X)=T_{\bar{n}}(x, \bar{X})=1 \mathrm{im}$ plies conditions $i$ ) and $i$ ) of Definition 3. Then, from Proposition 7 and Corollary 1 , we have $T_{n}(x, X)=1$ and $\left.T_{\bar{n}}(x, \bar{X})=1 \Rightarrow i i i\right)$ and $\left.i v\right)$. 


\subsection{End of the Proof of Theorem 2}

Proof of Theorem 2: Following Definition 3, a simple voxel obviously satisfies the three conditions of Theorem 2. Now, from Proposition 2, a voxel which satisfies the three conditions of Theorem 2 is such that $T_{n}(x, X)=T_{\bar{n}}(x, \bar{X})=1$. Finally, from Proposition 6 , if $T_{n}(x, X)=T_{\bar{n}}(x, \bar{X})=1$ then $x$ satisfies the four conditions of Definition 3.

\section{Conclusion}

The digital linking number allows us to formalize in a comprehensive way the characterization of 3D simple points for the complementary adjacency couples $(6,26)$ and $(26,6)$. The new theorem which is proved here shows the usefulness of the linking number in order to prove new theorems which involve the digital fundamental group in $\mathbb{Z}^{3}$. Now, even if the linking number is well defined for $(n, \bar{n}) \in\{(6+, 18),(18,6+)\}$, it has not been used yet to provide a characterization of $3 \mathrm{D}$ simple points, similar to Theorem 2, for the latter couples of adjacency relations. This, because an open question remains about the existence of a simple closed curve, analogue to the curves $c$ of Lemma 1, in this case.

\section{References}

Ber94. G. Bertrand. Simple points, topological numbers and geodesic neighborhoods in cubics grids. Patterns Recognition Letters, 15:1003-1011, 1994. 29

BM94. G. Bertrand and G. Malandain. A new characterization of three-dimensional simple points. Pattern Recognition Letters, 15:169-175, February 1994. 32, 33

FM00. S. Fourey and R. Malgouyres. A digital linking number for discrete curves. In Proceedings of the 7th International Workshop on Combinatorial Image Analysis (IWCIA'00), pages 59-77. University of Caen, July 2000. 27, 28, 30

Kon88. T. Y. Kong. Polyhedral analogs of locally finite topological spaces. In R. M. Shortt, editor, General Topology and Applications: Proceedings of the 188 Northeast Conference, Middletown, CT (USA), volume 123 of Lecture Notes in Pure and Applied Mathematics, pages 153-164, 1988. 29

Kon89. T. Y. Kong. A digital fundamental group. Computer Graphics, 13:159-166, 1989. 27, 29, 31

KR89. T. Y. Kong and Azriel Rosenfeld. Digital topology : introduction and survey. Computer Vision, Graphics and Image Processing, 48:357-393, 1989. 27

Mor81. D. G. Morgenthaler. Three-dimensional simple points : serial erosion, parallel thinning, and skeletonization. Technical Report TR-1005, Computer vision laboratory, Computer science center, University of Maryland, February 1981. 27,28

Rol. Dale Rolfsen. Knots and Links. Mathematics Lecture Series. University of British Columbia. 30

TF82. Y. F. Tsao and K. S. Fu. A 3d parallel skeletonwise thinning algorithm. In Proceeddings, IEEE PRIP Conference, pages 678-683, 1982. 31 